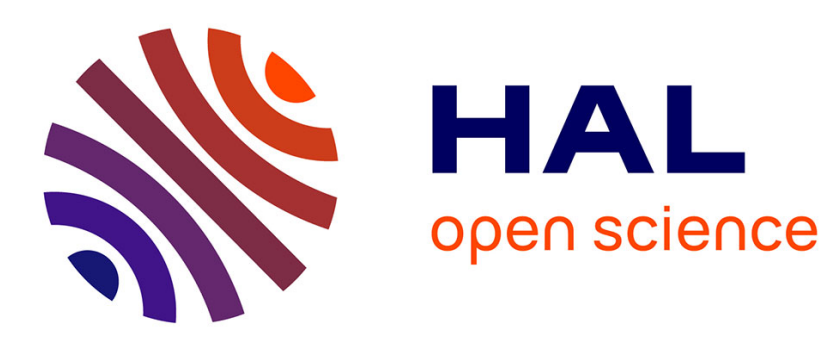

\title{
Fragilisation de la définition collective du travail bien fait et emprise des indicateurs d'activité
}

Marc Loriol

\section{To cite this version:}

Marc Loriol. Fragilisation de la définition collective du travail bien fait et emprise des indicateurs d'activité. Nouvelle revue de psychosociologie, 2020, 10.3917/nrp.029.0063 . halshs-02864044

\section{HAL Id: halshs-02864044 \\ https://shs.hal.science/halshs-02864044}

Submitted on 10 Jun 2020

HAL is a multi-disciplinary open access archive for the deposit and dissemination of scientific research documents, whether they are published or not. The documents may come from teaching and research institutions in France or abroad, or from public or private research centers.
L'archive ouverte pluridisciplinaire HAL, est destinée au dépôt et à la diffusion de documents scientifiques de niveau recherche, publiés ou non, émanant des établissements d'enseignement et de recherche français ou étrangers, des laboratoires publics ou privés. 


\title{
Fragilisation de la définition collective du travail bien fait et emprise des indicateurs d'activité
}

(Nouvelle revue de psychosociologie. 1 (29), 2020, p 63-73)

Marc Loriol

\begin{abstract}
Résumé
La mise en place d'indicateurs d'activité dans les organisations publiques ou privées comme mode de management produit une réduction du sens du travail et une fragilisation des règles de métier. Cela induit des résistances de la part des salariés et des collectifs de travail. Pourtant, dans certains cas, le chiffre peut devenir une référence importante pour les salariés. Les cas de deux policiers d'une brigade de police de proximité et d'une scène de musiques actuelles (SMAC), issus de deux recherches qualitatives (observations et entretiens), illustrent le lien entre l'attachement aux résultats quantitatifs d'activité et la difficulté à construire ensemble un sens positif et valorisant au travail. La comparaison avec d'autres collectifs de travail dans des organisations du même type permet de mettre en évidence les limites et les risques d'une valorisation de soi par les chiffres.
\end{abstract}

\section{Mots-clés}

Police, salles de concert, nouveau management publique, indicateur d'activité, beau travail, souffrance.

\begin{abstract}
The implementation of performance measurement systems in public or private organizations as a management method reduces work's meaning and weakens the rules of the trade. This induces resistance from employees and work collectives. However, in some cases, the figure can become an important reference for employees. The case of two policemen from a community police brigade and a scene from contemporary music (SMAC), resulting from two qualitative research (observations and interviews), illustrate the link between attachment to quantitative activity measurement and the difficulty of building together a positive and rewarding sense of work. Comparison with other work collectives in similar organizations highlights the limits and risks of self-worth through figures.
\end{abstract}

\section{Keywords}

Police, concert halls, new public management, activity indicator, nice job, suffering.

Le poids croissant accordé aux intérêts à court terme des actionnaires par rapport aux autres «parties prenantes » de l'entreprise (Gaulejac et Hanique, 2015) comme la politique du chiffre dans la fonction publique (Loriol, 2018) se traduisent par le passage de la demande de «bien faire » son travail (en fonction de normes plus ou moins partagées) à l'exigence de «faire plus » (que les autres, que l'an passé, etc.) du fait d'impératifs extérieurs à l'activité (rentabilité, compétitivité, cours boursiers, déficit public, etc.). Bien faire son travail, c'est se conformer à des normes et des règles définies localement et reconnues et appréciées par les pairs et la hiérarchie de proximité. Cela suppose la coopération, les débats sur le travail, la reconnaissance des efforts et des savoir-faire. À l'inverse, «faire plus » implique la concurrence et la désignation de «perdants » : tout le monde ne peut pas être le «premier»ni faire mieux que les autres! 
Défendre le travail «bien fait » est donc une façon de résister collectivement à l'emprise de l'organisation managériale, à la politique du chiffre. Dès lors, il peut sembler paradoxal que certains salariés cèdent parfois à l'emprise des chiffres alors même que les directions ne poussent pas explicitement dans ce sens ou que d'autres salariés soumis aux mêmes contraintes résistent plus fermement aux injonctions managériales. Pourtant, dans mes recherches sur les policiers (menées en 2003-2005 avec Valérie Boussard et Sandrine Caroly) puis sur les scènes de musiques actuelles (menées en 2013-2015 avec Line Spielmann : Loriol et Spielmann, 2015 ; Loriol, 2017 ${ }^{1}$ ), j'ai pu observer cette emprise du chiffre acceptée, voire recherchée par certains.

Prenant au sérieux l'idée de sociologie clinique, l'étude détaillée de comportements particuliers et atypiques replacés dans leurs contextes sociaux et organisationnels respectifs est un moyen de mieux comprendre comment le chiffre peut se substituer à d'autres formes de légitimation. Comment l'organisation formelle et informelle du travail façonne des attitudes psychiques spécifiques. Ces deux exemples permettent alors d'illustrer plus largement les liens entre la difficulté à valoriser collectivement le travail bien fait, la souffrance et l'adhésion in fine aux chiffres.

Dans une étude comparative sur deux organisations (une Caisse d'allocation familiale et une entreprise commerciale privée), Valérie Boussard (2001) avait montré comment les salariés interrogés présentaient leur activité en se référant constamment à un ou deux indicateurs (respectivement le «stock-retard » dans le traitement des dossiers à la Caisse d'allocations familiales et le taux de croissance annuel du bénéfice dans l'entreprise) bien que ces chiffres soient perçus comme réducteurs. Ces indicateurs prégnants servaient d'arguments pour se convaincre de la légitimité de l'organisation dans un contexte où les salariés avaient le sentiment que leur travail n'était pas reconnu. Le «stock-retard» dans le traitement des dossiers «attestait» ce que les agents produisaient tandis que le taux de croissance annuel rassurait les salariés sur la pérennité de l'entreprise.

Le travail bien fait ou «beau travail » n'existe pas a priori, mais dépend de règles élaborées en commun, d'échanges et même de conflits sur la qualité (Clot, 2010). L'absence de discussion collective sur la nature de ce qu'est le bon travail peut alors engendrer le repli sur le chiffre comme critère de remplacement pour évaluer l'activité. L'exemple des policiers montre comment ceux qui se sentent les moins légitimes s'emparent des indicateurs d'activité mobilisés par la hiérarchie (partie 1) tandis que leurs autres collègues intègrent a minima l'exigence de résultats chiffrés, voire s'y opposent. De plus, être fier du travail réalisé suppose de poser des limites, d'accepter de ne pas pouvoir tout faire. Là encore, le rôle du collectif est de définir les priorités et le niveau suffisant d'investissement pour considérer le travail comme bien fait, sinon les salariés peuvent s'épuiser à poursuivre un objectif inatteignable et finissent par voir dans le chiffre, comme dans le cas d'une scène de musique actuelle, un substitut à l'accord sur ce que doit être un concert réussi (partie 2). Dans les deux cas, l'absence de «jugements de beauté et d'utilité » partagés sur le travail amène les sujets à se tourner vers des indicateurs exogènes à leur activité pour tenter de se légitimer. Mais la reconnaissance par les chiffres est-elle suffisante pour soutenir le sens de l'action? Ne risque-t-elle pas au contraire d'affaiblir les collectifs et les valeurs de métier qui permettent justement d'étayer la

\footnotetext{
${ }^{1}$ Ces recherches sur quatre commissariats et trois scènes de musique actuelles s'appuient sur des observations in situ (des brigades de police-secours et de proximité et d'organisation de concerts et de réunions dans les scènes de musiques actuelles) et des entretiens semi-directifs (61 avec des policiers et 25 dans les scènes de musiques actuelles).
} 
construction d'un sens positif au travail et aux efforts consentis ? À l'inverse, là où l'action fait sens, le chiffre revêt moins d'importance. Il y a donc un lien circulaire entre la difficulté à définir collectivement ce qu'est le beau travail et l'emprise des dispositifs gestionnaires d'évaluation quantitative de l'activité (partie 3).

\section{Une adhésion enthousiaste par deux agents de police de proximité en quête de légitimité}

Lors de l'étude auprès de policiers de sécurité publique, j'ai observé que deux des agents d'une brigade de police de proximité étaient les seuls, dans les quatre commissariats étudiés, à attacher une grande importance aux résultats quantitatifs imposés par la «politique du chiffre ». Au cours des observations, $\mathrm{j}$ 'ai pu les voir à plusieurs reprises consulter les résultats d'activité par brigade (affichés dans la partie réservée à l'administration du commissariat), ce que ne faisaient pas les autres gardiens de la paix. De même, j'ai pu assister à un épisode étonnant où les deux agents de cette brigade avaient failli à la mission qui leur avait été confiée pour avoir passé trop de temps à verbaliser des véhicules mal garés en cours de route ! La police avait été appelée par des riverains au sujet d'un garagiste qui encombrait la chaussée avec des véhicules en réparation et des épaves. Deux véhicules d'enlèvement avaient été convoqués et, comme ce garagiste avait la réputation d'être irascible, voire violent, une protection policière avait été demandée. Alors que les deux gardiens de la paix se dirigeaient vers le garage en question, ils passèrent par une place où se trouvaient un grand nombre de véhicules en infraction. Ravis comme des promeneurs ayant déniché un coin à champignons, ils se mirent à verbaliser et oublièrent le rendez-vous. Quand ils s'en souvinrent, il était trop tard et les dépanneuses requises étaient parties. De retour au commissariat, ils se firent sermonner par le chef de brigade, contacté entre temps par l'un des dépanneurs mécontents.

La police de proximité, en France, n'a jamais obtenu le soutien hiérarchique et politique nécessaire à sa légitimation (Loriol, 2008). Jean-Pierre Chevènement, qui l'a mise place en 1997, l'a soutenue du bout des lèvres et ces brigades ont été partiellement supprimées par Nicolas Sarkozy en 2003. Sur les quatre commissariats étudiés, seuls deux avaient, au moment de l'étude, conservé un service de proximité. Ayant une position dévalorisée et des missions mal définies, considérés comme des «bouche-trous » (et non pour leur rôle particulier de contact avec la population), ces policiers voyaient dans le chiffre un moyen de démontrer malgré tout leur valeur, de prouver leur utilité et leur activité. Ils agissaient alors comme des «verbalisators » (jeu de mot local qui associe l'image du robot implacable Terminator et l'idée de verbaliser à tort), enfreignant les règles implicites à l'œuvre dans les autres brigades (les amendes infligées devant être liées au comportement de l'usager afin de laisser aux policiers une marge de négociation). Tout en défendant leur image de soi, ces policiers s'éloignaient donc plus encore de leurs collègues, se rendant plus encore dépendants des résultats chiffrés, dans une sorte de cercle vicieux.

La politique du chiffre déstabilise en effet surtout les brigades les plus fragiles, celles qui font moins bien que les autres ou se sentent moins reconnues. Alors que les brigades qui fonctionnent le mieux continuent de garder du temps pour se consacrer à ce qui représente à leurs yeux le «vrai travail policier », dans les autres, l'obligation de faire du chiffre remet en cause les significations locales de l'activité (seul compte ce qui peut être compté). Les pressions sur les chiffres accentuent également la concurrence entre policiers et brigades au détriment de la coopération. Dans les brigades qui font moins de chiffre, la pratique consistant à «donner » des timbres-amendes à ceux qui sont en dessous de la moyenne décline, tandis que BAC (brigade anti-criminalité avec des gardiens de la paix souvent en civil et spécialisés 
dans les flagrants délits) et brigades de police-secours rechignent à coopérer et à partager les « belles affaires ». Du coup, dans les brigades où la légitimité policière est la moins assurée, où le «vrai travail policier» semble inaccessible, comme dans les brigades de police de proximité ou la police municipale, il est parfois possible d'observer un certain repli sur les chiffres comme façon de se rassurer sur sa valeur : certes, l'on n'arrête pas des « voyous », on ne fait pas de «belles affaires », mais au moins nos résultats sont bons !

D'autres stratégies de revalorisation de soi dans cette brigade de proximité ont pu être observées, comme chez ce jeune policier qui avait une connaissance encyclopédique de l'histoire de la grande délinquance dans sa circonscription (et en faisait étalage devant le sociologue en observation), alors même que son travail quotidien était aux antipodes de la confrontation avec cette grande délinquance. Dans la même brigade de proximité, j'ai également longuement suivi deux autres gardiens de la paix, une femme qui a été auparavant la seule femme affectée en BAC, ce qui lui assure une certaine crédibilité, et un jeune homme sur qui elle a de l'influence. «Nous formons un couple professionnel », déclare-t-elle le premier jour d'observation. De fait, ils partagent une vision plus positive de la police de proximité et la policière envisage même de passer son BAFA (Brevet d'aptitude aux fonctions d'animateur) pour développer ses activités avec les jeunes. "Mais ne le dites pas à mes collègues, ils ne comprendraient pas », précise-t-elle après cet aveu. Pour eux, le contact avec les habitants est à la fois un enrichissement dans l'activité relationnelle et un moyen d'avoir une activité policière plus efficace : en se mêlant à la population et en gagnant la confiance des habitants, ils peuvent voir et apprendre plus de choses pour réaliser de « belles affaires ». C'est le sens et la qualité des interventions qui comptent et pas leur quantité. La brigade de proximité où étaient affectés les deux gardiens de la paix amateurs de chiffre se caractérisait ainsi par l'absence de collectif, chacun cherchant dans son coin (ou avec un collègue tout au plus) des façons de se revaloriser, mais sans pouvoir les partager avec les autres.

Dans les brigades de roulement étudiées (il y en a trois par commissariat), l'obligation de faire au moins les chiffres moyens du commissariat conduit à des attitudes diverses. Dans celles qui ont d'emblée les «bons » résultats sans avoir à courir après les chiffres, la recherche des «belles affaires » reste la préoccupation dominante. Quand la ville est trop tranquille et que l'ennui s'installe, les équipages peuvent décider de faire du contrôle routier ou de faire « la chasse aux petits shiteux » (qui ont quelques grammes de drogue sur eux), moins pour faire du chiffre que pour casser la routine. Par contre, pour les brigades qui se trouvent au-dessous de la moyenne, les résultats deviennent un objet de conflit récurrent avec la hiérarchie : chef de brigade qui impose des contrôles réguliers à heure fixe et commissaire qui « met la pression » sur le chef de brigade. Le sentiment de perte de contrôle sur l'activité et le sens du travail, l'impression de ne pas être reconnu par les supérieurs se traduisent par un malaise et un désengagement au sein des équipes, mais sans qu'il n'y ait pour autant un réinvestissement subjectif sur le chiffre, symbole de l'injonction hiérarchique illégitime et contre laquelle il $<$ faut résister. Enfin, dans une des BAC observées, Valérie Boussard a noté que les gardiens de la paix s'intéressaient à leurs résultats pour une raison particulière: leur objectif était d'atteindre le plus tôt possible les résultats mensuels attendus afin de pouvoir ensuite se consacrer pleinement à de «belles affaires » demandant plus de temps et de préparation. Pour la plupart des policiers suivis ou interrogés, et surtout les plus anciens, il y a donc bien des formes de résistance, variables d'une brigade à l'autre, à la politique du chiffre au nom des valeurs de métier et de l'autonomie de ceux qui sont sur le terrain.

\section{Une adhésion résignée dans une scène de musiques actuelles (SMAC)}


Dans une autre recherche, sur les salariés d'une salle de spectacle dédiée aux musiques actuelles (parmi les trois étudiées), appelée ici la Portée, toutes les personnes interrogées reconnaissaient que la réussite d'un concert, comme la qualité d'une action artistique et culturelle, ne peut pas se mesurer à l'aune de la réussite commerciale. Faire venir des groupes déjà connus qui plaisent au plus grand nombre n'est pas leur objectif. Le but affiché étant plutôt de faire connaître des musiciens novateurs et exigeants, de faire venir des publics qui ne sont pas conquis d'avance, de favoriser la création et l'échange culturel, de construire des liens avec les groupes locaux, etc.

Pourtant, du fait des divergences quant aux types de musiques et d'initiatives à promouvoir et en l'absence de critères partagés sur ce que devrait être un «beau concert », le nombre de places payantes vendues était devenu une sorte de plus petit dénominateur commun dans les évaluations faites ex post, malgré la contradiction avec les valeurs affichées (ne pas faire de la musique «commerciale »). Le fait que la salle ait fait l'objet d'investissements importants de la part de la municipalité et de subventions du ministère de la Culture, le sentiment d'être payé pour réaliser sa passion et l'engagement dans des missions valorisantes poussaient les salariés à vouloir donner en retour le meilleur d'eux-mêmes et le nombre de spectateurs payants était devenu un critère imparfait mais consensuel de «réussite »- même si un succès commercial ne garantissait pas un «beau concert».

Il n'y a pas d'accord à la Portée sur le type de musique qui motive les salariés et les bénévoles, sur celle qui justifie le mieux l'action culturelle envisagée. Ainsi, le «meilleur souvenir de concert » (selon l'une des questions de l'entretien) est différent pour chacun. Cela peut empêcher la passion d'être vécue pleinement et sereinement et venir en quelque sorte gâcher le plaisir si l'on a le sentiment qu'il n'est pas partagé, comme l'illustrent les exemples suivants. Le premier concerne un concert de hardcore pour lequel certains salariés et bénévoles étaient particulièrement enthousiastes (notamment du fait de la présence d'un groupe mythique). Mais il s'agit d'un style dont l'audience est restreinte. Même avec un dispositif qui réduisait la taille habituelle de la salle, le public apparaissait clairsemé (150 personnes pour près de 400 places). Le concert, bien qu'apprécié, a alors été vécu sur le registre de la culpabilisation: l'opération n'est pas «rentable», n'atteint pas le public souhaité... Comme le dit un bénévole qui a pourtant «adoré » le concert, « ça faisait tout triste de voir la salle comme ça, ça a cassé l'ambiance ».

Le deuxième exemple a trait aux concerts de jazz : appréciés par une partie seulement de l'équipe de direction, ce style musical semble en outre être le plus coûteux en raison des cachets demandés par les artistes et du public restreint. Du fait de sa reconnaissance culturelle plus établie, le jazz est parfois exclu du périmètre des «musiques actuelles» (les financements publics devant aller aux musiques qui en ont le moins). Là aussi, il peut y avoir un sentiment de culpabilité qui vient gâcher le plaisir : les amateurs de jazz ne se sentent pas tout à fait légitimes pour imposer un style musical qui coûte de l'argent à la structure sans emballer la majorité des équipes et du public, mais qui peut paraître relever évidemment de la mission «culturelle » et « artistique » de la Portée.

Cette absence de débat et d'accord sur le travail produit tout un ensemble de micro-conflits ou d'incidents qui restent dans le non-dit et révèlent le faible niveau de confiance en soi des équipes, malgré les réussites, comme le montre un troisième exemple : le concert d'un groupe connu ayant fait ses débuts des années auparavant à la Portée. Lors des balances et des répétitions du matin, le régisseur studio tente de passer aux musiciens des enregistrements de groupes amateurs locaux afin de solliciter leur avis. Le programmateur (qui doit aussi ce jour- 
là remplacer le barman en arrêt-maladie) fait barrage et s'énerve contre une pratique qu'il juge «peu professionnelle». Le programmateur s'énervera aussi peu après contre les bénévoles, leur reprochant de «s'amuser» plutôt que de «travailler». De plus, lors du montage du dispositif scénique (construit autour d'un échafaudage de chantier permettant aux musiciens de passer d'un étage à l'autre), l'équipe constate que le plafond de la scène est un peu plus bas que spécifié dans les plans. Une alternative doit être trouvée dans l'urgence. Le concert se passe très bien et est de qualité, les musiciens sont émus de revenir à la Portée, mais l'équipe n'a pas vécu ce concert aussi bien qu'elle l'aurait dû. Un des membres de l'équipe a même déclaré (apparemment sans avoir conscience du jeu de mot) : "Nous n'avons pas été à la hauteur ! »

La difficulté vient de ce que les missions des scènes de musiques actuelle sont potentiellement sans limites : favoriser l'innovation musicale; encourager les rapprochements entre styles musicaux et niveaux de professionnalisation ; faire preuve d'excellence artistique ; élargir les publics ; faire venir à la culture et à des pratiques exigeantes de nouveaux publics ; être un vecteur d'intégration sociale, culturelle et économique pour des jeunes en difficultés mais sensibles à la musique, etc. Vouloir tout faire à la fois est impossible, comme le montrent les conflits quotidiens à la Portée qui résultent en fait de hiérarchisations différentes des missions culturelles : par exemple, quand le programmateur pense qu'il faut «protéger » les groupes reconnus des demandes de musiciens amateurs locaux soutenus par une autre partie de l'équipe ou quand le régisseur scène vient prendre au dernier moment dans les studios de répétition un équipement manquant, privant des groupes locaux de leur session réservée à l'avance.

Quand la légitimité d'une action ou les critères de qualité (ce qu'est un « beau » travail, un «bon » choix de style musical) ne sont pas évidents ou partagés par tous peut émerger la tentation de se rattacher à ce qui serait une sorte de plus petit dénominateur commun : le quantitatif, les chiffres, notamment financiers (nombre spectateurs payants, équilibre des comptes, etc.). D'où le double paradoxe évoqué par certains administrateurs ou responsables de la Portée : l'intérêt pour les résultats chiffrés dans une structure qui fonctionne plutôt sur d'autres valeurs et la «passion » au travail qui devient un «problème » (surinvestissement et épuisement). Cela est renforcé par l'injonction politique à rationaliser, rationner, contrôler et justifier de plus en plus les dépenses publiques. Les musiques actuelles sont arrivées après les grands projets institutionnels pour les musiques classiques et la composition contemporaine et ne s'identifient pas à ce modèle. Un nouveau modèle est à inventer, entre Opéra et IRCAM plutôt élitistes et fermés - d'un côté et variétés commerciales de l'autre. Mais entre ces deux extrêmes, il est difficile de savoir précisément vers quoi aller, comment évaluer la réussite, comment définir l'idéal à atteindre.

Par peur de générer des conflits ingérables, en raison de la passion qui les anime et du fait du fort charisme du directeur, les équipes tiennent le coup en allant vers le toujours plus (de concerts, d'activités annexes, d'innovations, etc.) jusqu'à ce que le corps ou l'esprit craque et se révolte. La crainte de voir éclater ce fragile équilibre expliquait la réticence à mettre ouvertement en débat les divergences et les contradictions qui n'apparaissaient que de façon épisodique à travers divers petits accrochages, vécus sur le registre interpersonnel plutôt qu'organisationnel.

Mais cette fuite en avant n'est pas une fatalité et les deux autres structures étudiées dans le domaine des musiques actuelles ont géré différemment les questions de l'engagement passionné dans l'activité et des contraintes budgétaires. Si, à la Portée, l'absence de consensus 
sur ce que doit être un concert et une action culturelle réussie a conduit les équipes à vouloir toujours en faire plus, tout en mesurant in fine la réussite au seul critère imparfait du nombre de place payantes, une situation différente a été observée au Rézo (nom fictif). Cette salle plus petite a fait le choix, quelques années avant l'étude, de ne plus demander le label SMAC trop exigeant et porteur d'injonctions contradictoires. Tous les membres de l'équipe partagent une même conception de ce que doit être un concert réussi. Spécialisée sur le hip-hop et les musiques du monde, cette salle cherche à connecter les deux genres musicaux tout en menant, en marge des concerts, des actions visant à rapprocher les musiciens professionnels des groupes amateurs et des jeunes du quartier (featuring, jam session, ateliers d'écriture avec des rappeurs, etc.) Les stars du domaine qui refusent de se prêter au jeu sont écartées de la programmation, même si elles garantissent un succès commercial. Alors qu'à la Portée les treize personnes interrogées ont cité des concerts différents comme meilleur souvenir professionnel, au Rézo les sept personnes interrogées ont toutes évoqué le même (qui avait réuni un groupe vétéran de musiques du monde et de jeunes rappeurs). Dans cette salle, les salariés se disent passionnés par leur travail, mais estiment qu'ils parviennent à préserver du temps pour leur vie personnelle car ils ne sont pas obligés de toujours en faire plus pour tenter d'atteindre un idéal inaccessible.

Dans une troisième salle (appelée ici l'ABC), une situation encore différente prévalait. Le directeur de la salle entretenait des relations complexes avec la municipalité assurant une partie des subventions et avec la DRAC (Direction régionale de l'action culturelle). Pour gérer cette situation, il assurait lui-même le rôle de programmateur, laissant peu de place à son équipe qui en retour se sentait démotivée et peu impliquée, en attente d'un poste plus intéressant. Les salariés du Rézo et de l'ABC présentaient donc deux formes opposées de résistance aux injonctions politiques et managériales pesant sur les SMAC : les premiers ont collectivement choisi, avec la direction, de renoncer au label SMAC pour mieux préserver une conception forte et partagée de l'action culturelle et artistique (ce qui était possible grâce à un soutien de la ville où la salle est située et différents partenariats avec des entreprises); les seconds se sont protégés par une forme de retrait et de désinvestissement.

\section{Le chiffre comme palliatif imparfait au « beau travail »}

Les deux gardiens de la paix en police de proximité et la Portée sont atypiques dans leur tentative de préserver leur engagement et leur passion tout en se rabattant sur le chiffre. Dans ces deux cas où une emprise du chiffre a été observée, celle-ci révèle une souffrance liée à l'affaiblissement ou à l'absence des collectifs de travail, une légitimité professionnelle mal étayée, de faibles capacités à construire et soutenir un sens fort et partagé pour l'activité. Mais dans le même temps, cette valorisation du chiffre, des résultats quantifiables - qui participe d'évolutions économiques et sociales plus large (financiarisation des entreprises, nouveau management public, etc.) - favorise la concurrence entre structures ou entre salariés (le benchmarking crée une compétition), fait apparaître artificiellement des «mauvais » (tout le monde ne peut pas être le premier) et accroît en retour la difficulté à construire ensemble d'autres critères d'évaluation du travail bien fait, plus qualitatifs et fondés sur des normes communes. Le risque est de susciter un surinvestissement dans le travail, des frustrations et des déceptions du fait d'idéaux mal définis, donc peu atteignables, et de fragiliser tant des collectifs que des individus (épuisement, dépression, narcissisme...). La passion et l'engagement au travail sont des constructions collectives complexes qui doivent être régulées collectivement. Le chiffre devient une forme de palliatif imparfait qui imprègne plus ou moins les représentations des salariés et fragilise en retour les régulations collectives dans un cercle 
vicieux qui peut toucher même des secteurs qui a priori échappent ou résistent à l'emprise managériale.

Ce palliatif est insuffisant pour transformer durablement les contraintes et difficultés en sources de valorisation collective, en plaisir. Pour Hartmut Rosa (2018), la «force sociale », au sens de Durkheim, et le plaisir d'être ensemble découlent de ce qu'il appelle la « résonance », c'est-à-dire le sentiment de vibrer avec les autres et le monde, de participer à un projet commun, d'être en harmonie avec ce qui semble beau, bon et juste. La résonance dans le travail est différente de celle liée à d'autres formes de relations (amour, amitié...), car elle dépend de la médiation avec l'objet du travail (bien ou service produit). Cet objet est collectivement construit au double sens du terme, car il est fabriqué par l'intervention de plusieurs travailleurs et sa définition est structurée par des normes locales plus ou moins partagées. Qu'est-ce qu'un « beau travail », le «bien-être » ou l'intérêt des usagers, quand des contraintes extérieures à l'activité s'imposent par rapport à ces normes partagées et liées à une histoire propre à chaque collectif de travail ? Le travail perd de son sens, est moins porteur d'un sentiment d'auto-efficacité, de connivence avec les collègues et la hiérarchie de proximité sur le travail. Sans références partagées à l'objet du travail, les éventuelles marques de reconnaissance apparaissent purement instrumentales, vides de sens, incapables de justifier durablement les sacrifices consentis par passion dans le travail. À la Portée, d'ailleurs, le programmateur hyperactif fera une dépression et démissionnera après avoir été désavoué par la direction qui lui imposait le choix d'un groupe de rap qu'il jugeait peu créatif, mais qui connaissait à ce moment-là un fort succès commercial (toutes les places seront vendues en deux jours).

C'est pourquoi, dans les deux exemples évoqués, l'adhésion aux chiffres ne permet pas de supprimer la souffrance. Les indicateurs quantitatifs d'activité poussent au toujours plus, au dépassement de soi et des autres sans limites car sans objectifs intrinsèques et régulés localement, mais au contraire extrinsèques et liés à des intérêts extérieurs au collectif de travail (par exemple, réduire les déficits publics, faire de l'affichage politique, etc.). Non seulement les chiffres ne reflètent qu'une partie de l'activité, mais ils sont de plus porteurs d'une vision du travail propre aux décideurs (efficacité abstraite, rentabilité, polyvalence, etc.), de plus en plus coupés des réalités du terrain. Ils font donc difficilement sens en dehors de la seule motivation de la compétition et de la concurrence. Faire plus de «bâtons » (de chiffre, dans le langage policier) que les autres brigades ou faire plus d'entrées payantes que la salle de concert voisine ou que l'an passé ne sont pas des objectifs en soi. Ne se concentrer que sur les résultats conduit de plus à limiter le temps et les efforts consacrés à la réalisation d'un «beau travail» (intervenir sur des «belles affaires» en police-secours ou faire des concerts novateurs et exigeants dans les SMAC).

Ce qu'il y a de commun entre la Portée et les deux policiers de proximité et leurs collègues de la même brigade, c'est la crainte de mettre collectivement en débat le travail, l'absence de règles de métier partagées pouvant être opposées aux injonctions gestionnaires et un affaiblissement, voire une absence, des étayages collectifs. Si cette situation n'est ici l'objet d'aucun projet délibéré, mais plutôt le produit contingent d'injonctions politiques contradictoires, d'autres organisations ont bien perçu la force de ce processus pour affaiblir les résistances potentielles et favoriser une adhésion au chiffre, au profit d'intérêts étrangers à ceux des collectifs de travail. Confronter des travailleurs atomisés et isolés à un idéal à la fois inatteignable et vide de significations socialement étayées est un moyen d'augmenter artificiellement l'engagement et l'adhésion à court terme, les conséquences à moyen terme 
étant assumées par la collectivité (assurance maladie, invalidité ou chômage) et de plus en plus par les victimes elles-mêmes à qui l'on reproche de coûter trop cher.

\section{Bibliographie}

Boussard, V. 2001. "Quand les règles s'incarnent. L'exemple des indicateurs prégnants », Sociologie du travail, 43, 533-551.

Clot Y. 2010. Le travail à cœur. Pour en finir avec les risques psychosociaux, Paris, La Découverte.

Gaulejac (de), V.; Hanique, F. 2015. Le capitalisme paradoxant. Un système qui rend fou, Paris, Le Seuil.

Loriol, M. 2008. «La légitimité de l'action policière et les contacts avec la population face aux réformes récentes de la sécurité publique », dans J.-M. Berlière, C. Denys, D. Kalifa et V. Milliot (sous la direction de), Métiers de police. Être policier en Europe, XVIII ${ }^{e}-X X^{e}$ siècle, Presses universitaires de Rennes, 275-286.

Loriol, M. 2017. "The collective regulation of SMAC workers' passion and involvement», Sociologia del Lavoro, 145, 168-183.

Loriol, M. 2018. «L'irrationalité du NMP : le cas de l'hôpital et de la police », La revue des conditions de travail, 8, 47-57.

Loriol, M. ; Spielmann, L. 2015. «Quand la passion s'emmêle. De l'investissement de soi à la souffrance dans une MJC, scène de musiques actuelles », dans M. Loriol et N. Leroux (sous la direction de), Le travail passionné, Toulouse, érès, 117-151.

Rosa, H. 2018. Résonance. Une sociologie de la relation au monde, Paris, La Découverte. 